\title{
Impact of an antimicrobial stewardship in a 126-bed community hospital with close communication between pharmacists working on post-prescription audit, ward pharmacists, and the antimicrobial stewardship team
}

Satoshi Nakamura ${ }^{1,2}$, Takashi Arima ${ }^{1,3}$, Ryoichi Tashiro ${ }^{1,4}$, Satomi Yasumizu ${ }^{1,5}$, Hayato Aikou², Emi Watanabe ${ }^{2}$, Takashi Nakashima², Yuho Nagatomo², Ikuyo Kakimoto ${ }^{2}$ and Toshiro Motoya ${ }^{2 *}$ (i)

\begin{abstract}
Background: Antimicrobial stewardship (AS) is defined as coordinated interventions to improve and measure the appropriate use of antimicrobial agents. However, available resources for AS differ depending on the size of the clinical setting. Therefore, AS programs based on guidelines need to be selected in order to implement AS in smallto medium-sized hospitals. The present study compared the impact of AS in a 126-bed community hospital between pre- and post-AS periods.

Methods: The present study was retrospectively performed by selecting data on eligible patients from electronic medical records stored in the central database of the hospital. The roles of the AS team included weekly rounds and recommendations on the appropriate use of antimicrobials, and pharmacists working on post-prescription audits and pharmaceutical care at the bedside closely communicated with the AS team to assist with its implementation. As process measurements, the order rate of culture examinations, the conducting rate of deescalation, antimicrobial use density (AUD), days of therapy (DOT), and the AUD/DOT ratio of carbapenems and tazobactam-piperacillin (TAZ/PIPC) were measured. Thirty-day mortality and recurrence rates were examined as clinical outcomes.
\end{abstract}

* Correspondence: motoya@tarumizumh.jp

${ }^{2}$ Division of Pharmacy, Tarumizu Chuo Hospital, Tarumizu Municipal Medical Center, Tarumizu, Japan

Full list of author information is available at the end of the article

C C The Author(s). 2021 Open Access This article is licensed under a Creative Commons Attribution 4.0 International License, which permits use, sharing, adaptation, distribution and reproduction in any medium or format, as long as you give appropriate credit to the original author(s) and the source, provide a link to the Creative Commons licence, and indicate if changes were made. The images or other third party material in this article are included in the article's Creative Commons licence, unless indicated otherwise in a credit line to the material. If material is not included in the article's Creative Commons licence and your intended use is not permitted by statutory regulation or exceeds the permitted use, you will need to obtain permission directly from the copyright holder. To view a copy of this licence, visit http://creativecommons.org/licenses/by/4.0/. The Creative Commons Public Domain Dedication waiver (http://creativecommons.org/publicdomain/zero/1.0/) applies to the data made available in this article, unless otherwise stated in a credit line to the data. 
(Continued from previous page)

Results: A total of 535 patients (288 in the pre-AS period and 247 in the post-AS period) were enrolled in the present study. The recommendation rate to prescribers significantly increased $(p<0.01)$ from $10.4 \%$ in the pre-AS period to $21.1 \%$ in the post-AS period. The order rate of culture examinations increased from 56.3 to $73.3 \%(p<$ 0.01). The conducting rate of de-escalation increased from 10.2 to $30.8 \%(p<0.05)$. The AUD of carbapenems and TAZ/PIPC significantly decreased $(p<0.05)$. The DOT of carbapenems $(p<0.01)$ and TAZ/PIPC $(p<0.05)$ also significantly decreased. The AUD/DOT ratio of carbapenem significantly increased from 0.37 to 0.60 ( $p<0.01$ ). Thirtyday mortality rates were 11.2 and $14.2 \%$, respectively, and were not significantly different. The 30-day recurrence rate significantly decreased $(p<0.05)$ from 14.7 to $7.5 \%$.

Conclusions: The implementation of AS in this hospital improved the appropriate use of antimicrobials without negatively affecting clinical outcomes. These results may be attributed to close communication between pharmacists working on post-prescription audits and pharmaceutical care at the bedside and the AS team.

Keywords: Antimicrobial stewardship, Antimicrobial stewardship team, Appropriate use of antimicrobials, Culture examination, De-escalation, Antimicrobial use density, Days of therapy, 30-day mortality, 30-day recurrence rate, Pharmacist

\section{Background}

Antimicrobial resistance (AMR) increases the difficulties associated with treating an infectious disease and is a serious threat to human health. It has resulted in longer hospital stays, higher hospital costs, and greater mortality $[1,2]$. The inappropriate use of antimicrobial agents is considered to be a major factor increasing AMR, and antimicrobial stewardship (AS) is defined as coordinated interventions to improve and measure the appropriate use of antimicrobial agents. AS has been shown to decrease antimicrobial use density (AUD), days of therapy (DOT), and in-hospital mortality and also contributes to cost savings [3-5].

AS is a medical stuff function that optimizes clinical outcomes while minimizing the unintended consequences of antimicrobial use, and includes the appropriate selection, dosing, route, and duration of antimicrobial therapy [6]. Recommended core members of the AS team (AST) include an infectious diseases physician, a clinical pharmacist with infectious disease training, a clinical microbiologist, an information system specialist, and an infection control professional. Comprehensive AS programs (ASPs) that include a number of elements, such as interventions, optimization, microbiology and laboratory diagnostics, measurements, special populations, and education, have been proposed as therapeutic guidelines to combat $\operatorname{AMR}[6,7]$. On the other hand, the conditions under which AS is implemented differ based on local antimicrobial use and resistance. Furthermore, available resources including manpower, on-site microbiology laboratories, and AST organizations differ depending on the size of the institution or clinical setting. According to a nationwide survey conducted by the Japanese Society of Chemotherapy, small- to medium-sized hospitals had fewer on-site microbiology laboratories, AST organizations, pharmacists and collaborations with ward pharmacists than large-sized hospitals $[8,9]$. Therefore, ASPs according to the guidelines need to be selected to implement AS in small- to medium-sized hospitals [6, 7]. To date, ASPs have been widely implemented in large-sized hospitals. Few studies have examined the implementation of AS in the small- to medium-sized hospitals [10-12], and the impact of AS on the use of antimicrobials and clinical outcomes in hospitals with finite resources is limited. Accordingly, further studies are needed on the strategies to improve the efficacy of AS in small- to medium-sized hospitals.

Tarumizu Chuo hospital is a 126-bed community hospital. The Infection Control Team (ICT) of the hospital include a physician (Infection Control Doctor: ICD), a clinical pharmacist without authorized infectious disease training, a nurse, and a clinical laboratory technologist, who concurrently have been members of AST since June 2019. Prior to the organization of AST, antimicrobial agents were administered at the discretion of the respective attending physicians. At that time, carbapenem or Tazobactam-Piperacillin (TAZ/PIPC) was administered to some cases as an empiric treatment for severe infectious diseases. Furthermore, antimicrobial agents were prescribed over long periods to some cases in which causative microorganisms were not identified. To improve these conditions, AST was organized in June 2019 , and started weekly rounds and prospective audits of antimicrobial use with direct interactions and feedback. Regarding the prospective audit of antimicrobial use, pharmacists including an AST pharmacist working on post-prescription audits also started to check medical records in detail every day and also collected information on the conditions of patients as ward pharmacists. Pharmacists other than the AST pharmacist closely communicated with the AST pharmacist and supported the implementation of AS. 
Therefore, the present study was performed to assess the impact of AS on the use of antimicrobials and clinical outcomes at this 126-bed community hospital with close communication between pharmacists working on post-prescription audit, ward pharmacists, and AST.

\section{Methods}

\section{Study design}

The present study was conducted at Tarumizu Chuo Hospital, Tarumizu Municipal Medical Center. Study periods were the pre-AS period (between July 1 and December 31, 2018) and post-AS period (between July 1 and December 31, 2019). Eligible patients were inpatients treated with specific antimicrobials, administered parenteral antimicrobials for more than 14days, with antimicrobial-resistant pathogens, and with a microbialpositive blood culture. Specific antimicrobials were meropenem (MEPM), imipenem/cilastatin (IPM/CS), TAZ/ PIPC, levofloxacin, cefepime, cefozopran, vancomycin, and linezolid. Antimicrobial-resistant pathogens included methicillin-resistant Staphylococcus aureus (MRSA), extended-spectrum $\beta$-lactamase-producing enterobacteria (ESBL-producing enterobacteria), carbapenem-resistant Enterobacteriaceae (CRE), vancomycin-resistant enterococci (VRE), multidrugresistant Acinetobacter spp. (MDRA), multidrugresistant Pseudomonas aeruginosa (MDRP). MDRA and MDRP were defined as strains that were resistant to carbapenems (minimum inhibitory concentration: MIC $\geq 16 \mathrm{mg} / \mathrm{L}$ ), fluoroquinolones ( $\mathrm{MIC} \geq 4 \mathrm{mg} / \mathrm{L}$ ), and amikacin (MIC $\geq 32 \mathrm{mg} / \mathrm{L}$ ) based on the criteria for MDR strains specified by the Law Concerning the Prevention of Infections and Medical Care for Patients with Infections by the Japanese Ministry of Health and Welfare [13]. Patients treated with specific antimicrobials and parenteral antibiotics for more than 14 days were extracted by the AST pharmacist, while those with antimicrobial-resistant pathogens and a microbialpositive blood culture were extracted by the AST clinical laboratory technologist before AST rounds.
AS implementation in the pre-AS period included the registration system of specific antimicrobials when used, the monthly monitoring of antimicrobial consumption, the monthly monitoring of antimicrobial resistance, and annual lectures for hospital staff and occasional discussions with an attending physician. These activities were continued in the post-AS period. Regarding the postprescription audit of antimicrobial use, dose adjustments based on the package inserts of medicines were implemented in the pre-AS period. Furthermore, careful checks of medical records, including prescriptions, laboratory test values, vital signs, and other conditions for eligible patients, were performed daily by pharmacists in the post-AS period. Pharmacists other than the AS pharmacist then closely communicated with the AST pharmacist and recommendations to prescribers from AST were implemented. Details on recommendations by AST to prescribers are shown in Table 1 and include the following: 1) Order of culture examinations, 2) Selection of antimicrobials, 3) Dose of antimicrobials, 4) Duration of antimicrobial therapy, 5) De-escalation, 6) Therapeutic drug monitoring, and 7) Others. Rounds by AST had not been implemented in the pre-AS period; however, weekly rounds were performed in the post-AS period. Ward pharmacists also collected information on the conditions of patients. They closely communicated with the AST pharmacist and supported the implementation of AS. Regarding the ward activity of pharmacists, the number of cases claiming the drug management guidance fee, one of the medical fees for pharmaceutical care by ward pharmacists, increased from 321 in the pre-AS period to 683 in the post-AS period. This increase reflected rounds being more frequently performed by ward pharmacists. The AST pharmacist typically integrated patient information on pharmacotherapy, corresponded for inquiries including the selection of antibiotics from prescribers, and made recommendations for the appropriate use of antibiotics where necessary. The frequency of recommendations was higher in the post-AS period than in the pre-AS period. Furthermore,

Table 1 Recommendations to prescribers from the antimicrobial stewardship team
1) Order of culture examinations
2) Selection of antimicrobials Identification of microbial pathogens and implementation of susceptibility tests before administration of parenteral antimicrobials.
Selection of antimicrobials for empirical initial antimicrobial therapy.
Changes in antimicrobials based on the results of susceptibility tests (not including de-escalation).
3) Dose of antimicrobials
Dose optimization based on the severity of disease and the renal function of patients.
4) Duration of antimicrobial therapy
5) De-escalation
Recommendation to discontinue antimicrobial therapy.
6) Therapeutic drug monitoring 
the AST pharmacist typically made hospital antibiograms and provided data to prescribers and other medical staff in order to ensure the appropriate use of antimicrobials in the post-AS period, in contrast to the pre-AS period.

The present study was retrospectively performed by selecting data on eligible patients from electronic medical records stored in the central database of the hospital.

\section{Monitoring of process measurements}

Regarding process measurements of the appropriate use of antimicrobials, the order rate of culture examinations to identify pathogens and the conducting rate of deescalation were examined and expressed as follows:

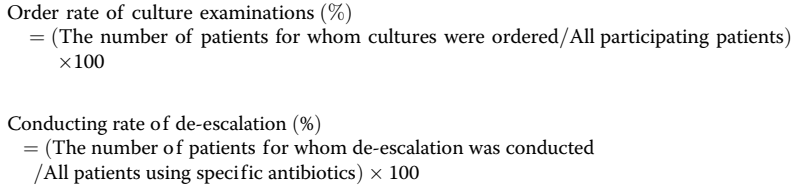

We also examined AUD, DOT, and the AUD/DOT ratio of carbapenems (MEPM and IPM/CS) and TAZ/ PIPC, which were more frequently used among the specific antimicrobials at this hospital. AUD and DOT were expressed as follows:

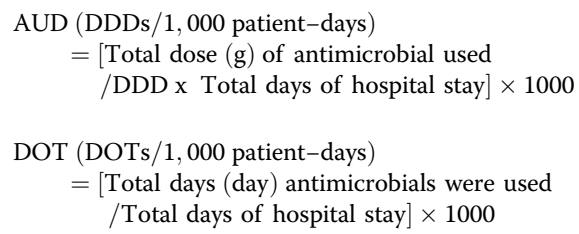

The defined daily dose (DDD) was the value defined by the World Health Organization.

\section{Clinical outcome}

We examined 30-day mortality and recurrence rates as the clinical outcomes of AS. The 30-day mortality rate was calculated as the number of patients who died within 30 days of the initiation of antimicrobial treatment divided by all participating patients. The 30-day recurrence rate was calculated as the number of patients with recurrence of the same disease within 30 days of discontinuing antimicrobial therapy divided by all participating patients.

\section{Statistical analysis}

Statistical analyses were performed using ystat2018.xls for Windows (Igakutosyosyuppan co., Ltd., Tokyo, Japan). Nominal variables of the two groups were compared using the Chi-squared test and continuous variables using the Mann-Whitney U-test. A $p$-values of $<$ 0.05 was considered to be significant.

\section{Ethics approval and consent to participate}

The present study was approved by the Ethics Committee of Tarumizu Chuo Hospital (No. 20-1). The Ethics Committee waived the need for informed consent since this study used anonymous, aggregate, retrospective data.

\section{Results}

\section{Patient characteristics}

A total of 535 patients were enrolled in the present study. The pre-AS and post-AS groups included 288 and 247 patients, respectively. The characteristics of patients enrolled in the present study are shown in Table 2. No significant differences were observed in age, sex, body weight, renal function, or infected organs between the groups. Among infected organs, the ratio of respiratory organs was the highest in both groups, but was not significantly different.

\section{AS activity}

Details on recommendations by the AST for prescribers are shown in Table 3. The total number of patients recommended was $30(10.4 \%)$ in the pre-AS period and 52 $(21.1 \%)$ in the post-AS period. The post-AS period showed a significantly higher rate $(p<0.01)$. The number of cases recommended were 30 and 68 , respectively. In the post-AS period, some patients received two or more recommendations. The acceptance rate of recommendations by prescribers was $93.3 \%(28 / 30)$ in the pre-AS period and $94.1 \%(64 / 68)$ in the post-AS period, which was not significantly different. The number of recommendations on the selection of antimicrobials increased the most in the post-AS period. The numbers of recommendations for de-escalation (7) and the duration of antimicrobial therapy (4) in the post-AS period increased from 0 cases in the pre-AS period.

\section{Process measurements}

The order rate of culture examinations for the identification of microbial pathogens and the conducting rate of de-escalation are shown in Fig. 1. The order rate of culture examinations significantly increased $(p<0.01)$ from $56.3 \%$ in the pre-AS period to $73.3 \%$ in the post-AS period. The conducting rate of de-escalation significantly increased $(p<0.05)$ from $10.2 \%$ in the pre-AS period to $30.8 \%$ in the post-AS period.

AUD, DOT, and the AUD/DOT ratio of carbapenems (MEPM and IPM/CS) and TAZ/PIPC in both periods are shown in Table 4. The AUD of carbapenem significantly decreased $(p<0.05)$ from $8.28 \pm 3.39$ in the pre-AS period to $3.67 \pm 1.73$ in the post-AS period. The AUD of TAZ/PIPC also significantly decreased $(p<0.05)$ from $15.05 \pm 5.91$ to $8.55 \pm 3.47$. The DOT of carbapenems significantly decreased $(p<0.01)$ from $21.62 \pm 7.23$ in the pre-AS period to $5.88 \pm$ 
Table 2 Patient characteristics

\begin{tabular}{|c|c|c|c|}
\hline & pre-AS period & post-AS period & $p$-value \\
\hline & $(n=288)$ & $(n=247)$ & \\
\hline Age (years) & $81.9 \pm 13.8$ & $82.9 \pm 12.2$ & 0.281 \\
\hline $\operatorname{Sex}(F / M)$ & $129 / 159$ & $111 / 136$ & 0.973 \\
\hline Body weight (Kg) & $47.0 \pm 13.8$ & $46.8 \pm 14.3$ & 0.435 \\
\hline $\mathrm{CCr}(\mathrm{mL} / \mathrm{min})$ & $46.2 \pm 30.7$ & $45.4 \pm 30.0$ & 0.363 \\
\hline \multicolumn{4}{|l|}{ Focus of infection } \\
\hline Respiratory & $148(51)$ & $152(62)$ & 0.212 \\
\hline Urinary tract & $75(26)$ & $44(18)$ & 0.068 \\
\hline Gastrointestinal and Intraabdominal & $38(13)$ & $27(11)$ & 0.479 \\
\hline Bone and soft tissue & $17(6)$ & $13(5)$ & 0.762 \\
\hline Others & $10(4)$ & $12(5)$ & 0.440 \\
\hline
\end{tabular}

Values are presented as means \pm SD or numbers (\%), $\mathrm{CCr}$ Creatinine clearance

Data were analyzed using the Mann-Whitney U-test or chi-squared test

1.76 in the post-AS period. The DOT of TAZ/PIPC also significantly decreased $(\mathrm{p}<0.05)$ from $20.93 \pm 8.76$ to $12.65 \pm$ 4.96. The AUD/DOT ratio of carbapenem significantly increased $(\mathrm{p}<0.01)$ from $0.37 \pm 0.07$ in the pre-AS period to $0.60 \pm 0.12$ in the post-AS period, while that of TAZ/PIPC decreased from $0.73 \pm 0.08$ to $0.67 \pm 0.07$, which was not significant.

\section{Clinical outcomes}

Thirty-day mortality and recurrence rates in the pre- and post-AS periods are shown in Fig. 2. Thirty-day mortality rates in the pre- and post-AS periods were 11.2 and $14.2 \%$, respectively, and were not statistically different. Thirty-day recurrence rates were 14.7 and $7.5 \%$, respectively, which was significantly $(p<0.05)$ lower in the post-AS period than that in the pre-AS period.

As described above, a decreases were observed in the AUD and DOT of carbapenem and TAZ/PIPC in the present study. Based on these results, improvements in the appropriate use of antimicrobials were achieved without negatively affecting clinical outcomes by AS in this hospital.

\section{Discussion}

Small- to medium-sized hospitals have finite medical resources. Therefore, the implementation of ASPs has to be performed by selecting items available, and their impact may be limited. An assessment of the impact of implementing ASPs is important. Mas-Morey et al.

Table 3 Recommendations to subscribers from the antimicrobial stewardship team

\begin{tabular}{|c|c|c|c|}
\hline & $\begin{array}{l}\text { pre-AS period } \\
(n=288)\end{array}$ & $\begin{array}{l}\text { post-AS period } \\
(n=247)\end{array}$ & $\begin{array}{l}p \text { - } \\
\text { value }\end{array}$ \\
\hline Order of culture examinations & 1 & 4 & \\
\hline Selection of antimicrobials & 4 & 26 & \\
\hline Dose of antimicrobials & 18 & 20 & \\
\hline Duration of antimicrobial therapy & 0 & 4 & \\
\hline De-escalation & 0 & 7 & \\
\hline Therapeutic drug monitoring & 3 & 5 & \\
\hline Others & 4 & 2 & \\
\hline Total recommendations (cases) & 30 & 68 & \\
\hline Applicable patients (persons) & 30 & 52 & \\
\hline Recommendation rate & $10.4(30 / 288)$ & $21.1(52 / 247)$ & $<0.01$ \\
\hline Acceptance rate of recommendation & $93.3(28 / 30)$ & $94.1(64 / 68)$ & 1.00 \\
\hline
\end{tabular}

Values are presented as numbers or \% (Number of recommended cases/All participating patients) or \% (Number of accepted cases/Number of recommended cases)

Data were analyzed using the chi-squared test 
(a)

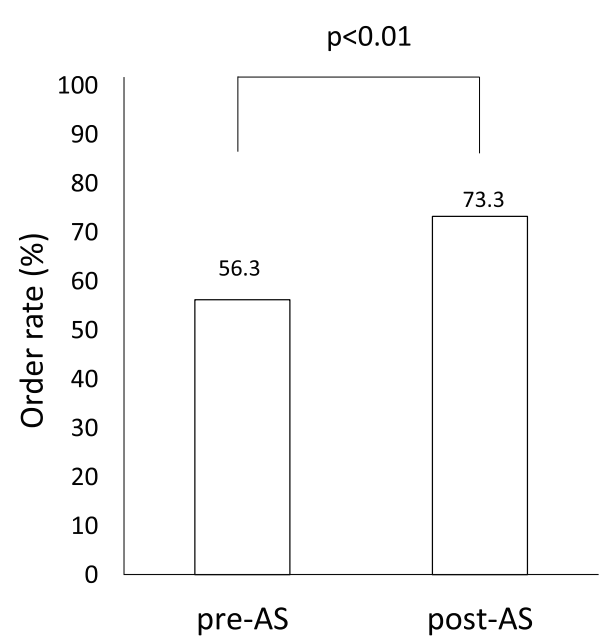

(b)

De-escalation

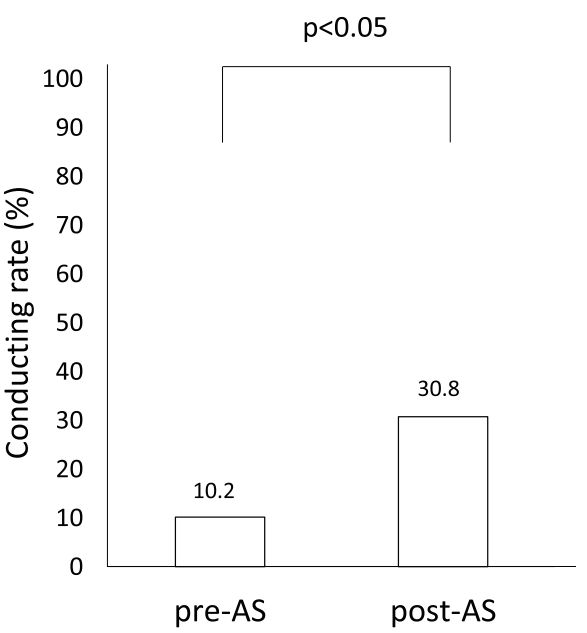

Fig. 1 Comparison of the order rate of culture examinations a and the conducting rate of de-escalation $\mathbf{b}$ between pre- and post-AS periods. The chi-squared test was used for statistical analyses

evaluated 28 studies implemented in 26 hospitals with < 500 beds and reported that interventions were not generally associated with significant changes in mortality or readmission rates; however, substantial cost savings were achieved, mainly due to the reduced use of antibiotics or the administration of cheaper antibiotics [10]. The present study compared the use of antimicrobials and its clinical outcomes between the pre- and post-AS periods at a 126-bed community hospital. The results obtained showed that the appropriate use of carbapenem and TAZ/PIPC improved and the 30-day recurrence rate significantly decreased; however, the 30-day mortality rate did not significantly change. Therefore, the implementation of AS in this hospital appeared to effectively improve the appropriate use of antimicrobials, similar to or more effectively than that reported for other hospitals.

The strategy of this study for ASP was to implement a prospective audit and feedback by AST closely communicating with ward pharmacists and pharmacists working on post-prescription audits. Dallit et al. highlighted 2 important core strategies for improving the impact of implementing ASPs: 1) a prospective audit with an intervention and feedback and 2) formulary restriction with preauthorization [6]. Anderson et al. recently reported that a post-prescription audit and review were a more feasible and effective strategy for AS in settings with limited resources and expertise than preauthorization [11]. Furthermore, Muraki indicated that a prospective audit and feedback were realistic for improving the implementation of AS in Japan, and close communication between the AST pharmacist and ward pharmacists was important for consecutive support of this implementation [14]. Doenberg et al. reported that the introduction of a weekly prospective audit and feedback was insufficient for effective ASP implementation, but achieved modest decreases in the utilization of antibiotics [15]. These findings are considered to support the strategy of the present study.

The present results showed that the recommendation rate to prescribers from AST significantly increased from 10.4 to $21.1 \%$ (Table 3 ), and the order rate of culture examinations also significantly increased from 56.3 to 73.3\% (Fig. 1a). These increases are considered to be a direct impact of early prospective audits and feedback to

Table 4 Changes in AUD, DOT, and the AUD/DOT ratio between pre- and post-AS periods

\begin{tabular}{|c|c|c|c|c|c|c|}
\hline & \multicolumn{3}{|l|}{ Carbapenem } & \multicolumn{3}{|l|}{ TAZ/PIPC } \\
\hline & pre-AS & post-AS & $\overline{p \text {-value }}$ & pre-AS & post-AS & $p$-value \\
\hline AUD & $8.28 \pm 3.39$ & $3.67 \pm 1.73$ & $<0.05$ & $15.05 \pm 5.91$ & $8.55 \pm 3.47$ & $<0.05$ \\
\hline DOT & $21.62 \pm 7.23$ & $5.88 \pm 1.76$ & $<0.01$ & $20.93 \pm 8.76$ & $12.65 \pm 4.96$ & $<0.05$ \\
\hline AUD/DOT & $0.37 \pm 0.07$ & $0.60 \pm 0.12$ & $<0.01$ & $0.73 \pm 0.08$ & $0.67 \pm 0.07$ & 0.15 \\
\hline
\end{tabular}

Values are presented as means \pm SD

AUD: Antimicrobial use density (DDDs/1000 patient-days), DOT: Days of therapy (DOTs/1000 patient-days)

Data were analyzed using the Mann-Whitney U-test 
(a)

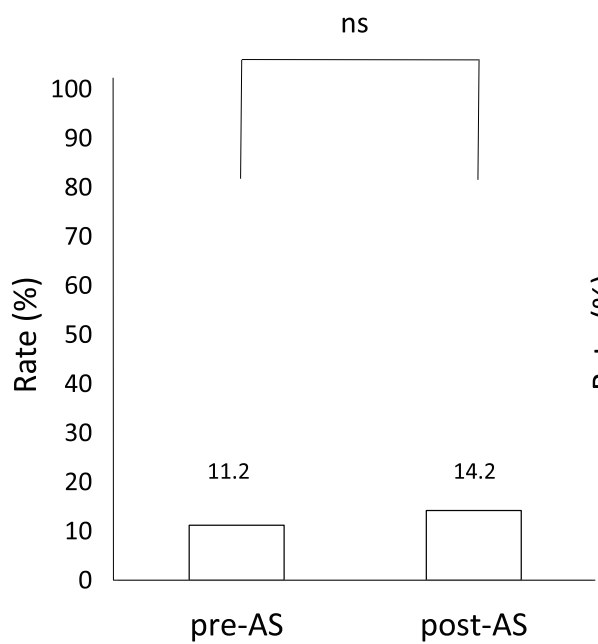

(b)

30-day recurrence rate

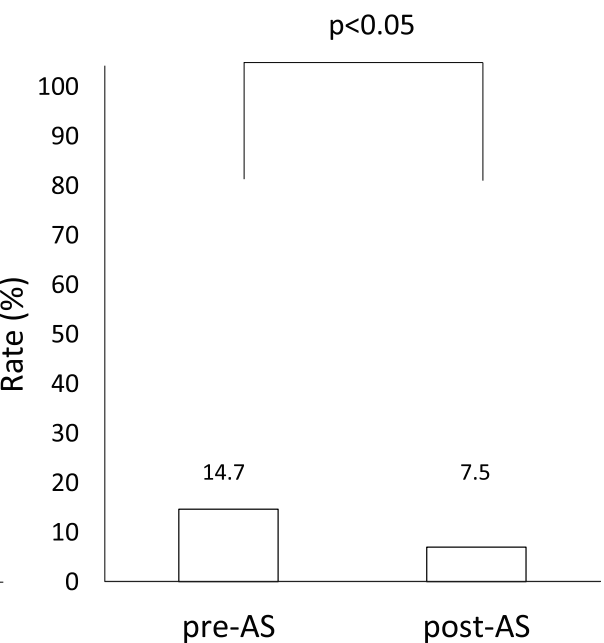

Fig. 2 Comparison of the 30-day mortality rate $\mathbf{a}$ and 30-day recurrence rate $\mathbf{b}$ between pre- and post-AS periods. The chi-squared test was used for statistical analyses

prescribers from AST and are attributed to close communication between AST and pharmacists, as described above.

The conducting rate of de-escalation significantly increased from $10.2 \%$ in the pre-AS period to $30.8 \%$ in the post-AS period (Fig. 1b). Culture examinations are necessary for the appropriate selection of antibiotics, including the implementation of de-escalation [16]. This increase was attributed to the significantly higher order rate of culture examinations. On the other hand, the order rate of culture examinations was $73.3 \%$, indicating that examinations were not ordered for the other $26.7 \%$ cases in the post-AS period, which is an issue that needs to be resolved. One approach to achieve improvements is to educate medical staff, including prescribers, about the importance of culture examinations through closer communication.

Decreases were observed in the AUD and DOT of carbapenem and TAZ/PIPC, respectively (Table 4), which indicated a reduction in the amount used and a shorter period of use of these antibiotics. The decreases observed in AUD of carbapenem and TAZ/PIPC in the present study were consistent with previous findings [4]. The decrease in the DOT of carbapenem was also consistent with previous findings [5, 17], whereas that of TAZ/PIPC was not. Yamada et al., [5] reported that the DOT of TAZ/PIPC significantly increased in the postASP period, which may be attributed to the use of TAZ/ PIPC as an alternative to carbapenems for severe infections and those with long-term antibiotic use. In the present study, the decrease in the DOT of TAZ/PIPC was mainly due to an increase in the conducting rate of de-escalation, and carbapenems and TAZ/PIPC were both typically switched to sulbactam/ampicillin or thirdgeneration cepharosporin. This appears to have been the reason for the differences between the present results and previous findings.

The AUD/DOT ratio of carbapenem significantly increased (Table 4). Carbapenems are some of the most important antibiotics for the treatment of severe or intractable infectious diseases. The daily dose of carbapenem was recently increased according to the worldwide recognition of the pharmacokinetic/pharmacodynamic (PK/PD) theory. Regarding MEPM, the administration of a daily dose of more than $3 \mathrm{~g}$ based on the PK/PD theory was reportedly safe and achieved more beneficial effects [18]. However, in this hospital, a daily dose of $1 \mathrm{~g}$ was generally administered according to the instructions on the package insert of medicines for common infectious diseases in the pre-AS period. In the present study, the ratio increased to close to 1.0, which may be attributed to closer communication and better feedback between AST and prescribers, including recommendations based on therapeutic guidelines for infectious diseases $[19,20]$. On the other hand, the AUD/DOT ratio of TAZ/PIPC did not significantly change. The average value of 0.73 in the pre-AS period was considered to be high because it was close to 1.0 and was maintained in the post-AS period.

In the present study, a significant decrease was observed in the 30-day recurrence rate; however, in a review of 28 studies, Mas-Morey et al. reported no significant reductions in readmission rates after the implementation of ASP [10]. The mediator(s) for the significant decrease in the recurrence rate in the present 
study remain unclear. One possibility may be longer hospital stays in the present study than in the studies reviewed by Mas-Morey et al. [10]. Average hospital stays in the present study were 38.2 days in the pre-AS period and 32.0 days in the post-AS period. In contrast, average or median hospital stays in the pre- and post-AS periods of some of the studies reviewed by Mas-Morey et al. were 3.40 and 2.69 days [21], 13.2 and 10.8 days [22], and 9.0 and 5.7 days [23], respectively. The longer continuous implementation of post-prescription audits and feedback to prescribers by pharmacists working on post-prescription audits in addition to careful checks of patients' conditions by ward pharmacists may have contributed to the decrease observed in the 30-day recurrence rate.

The present study had the following limitation: the relationship between clinical outcomes and AS was not clarified. Therefore, further progress in AS activity is necessary to improve clinical outcomes.

\section{Conclusions}

The implementation of AS at this 126-bed community hospital significantly increased recommendations on the appropriate use of antimicrobials from AST to prescribers. It also increased the order rate of culture examinations and the conducting rate of de-escalation. Furthermore, decreases were observed in the amount and periods of use of carbapenems and TAZ/PIPC without a negative impact on clinical outcomes, such as 30day mortality and recurrence rates, in the post-AS period from the pre-AS period. The beneficial effects of AS are considered to be achieved by close communication between pharmacists working on post-prescription audits and pharmaceutical care at the bedside and AST.

\begin{abstract}
Abbreviations
AMR: Antimicrobial resistance; AS: Antimicrobial stewardship;

AUD: Antimicrobial use density; DOT: Days of therapy; AST: Antimicrobial stewardship team; ASP: Antimicrobial stewardship program; ICT: Infection control team; ICD: Infection control doctor; TAZ/PIPC: TazobactamPiperacillin; MEPM: Meropenem; IPM/CS: Imipenem/cilastatin; DDD: Defined daily dose; PK/PD: Pharmacokinetic/pharmacodynamics
\end{abstract}

\section{Acknowledgements}

We acknowledge the contributions of Mr. Takafumi Fujisaki and Yuki Higashi.

\section{Authors' contributions}

SN, TA, RT, SY and TM contributed to the conception and design of the protocol, data collection, data analysis, and drafting of the manuscript. HA, EW, TN, YN and IK contributed to the conception, data collection, and drafting of the manuscript. All authors read and approved the final manuscript.

\section{Funding}

Not applicable.

\section{Availability of data and materials}

The datasets generated and/or analyzed during the current study are not publicly available because the transfer of data containing personal information outside of Tarumizu Chuo Hospital is not allowed.

\section{Declarations}

Ethics approval and consent to participate

Stated in the Methods section.

Consent for publication

Not applicable.

\section{Competing interests}

The authors declare that they have no competing interests.

\section{Author details}

${ }^{1}$ Infection Control Team and Antimicrobial Stewardship Team, Tarumizu Chuo Hospital, Tarumizu Municipal Medical Center, Tarumizu, Japan.

${ }^{2}$ Division of Pharmacy, Tarumizu Chuo Hospital, Tarumizu Municipal Medical Center, Tarumizu, Japan. ${ }^{3}$ Division of Urology, Tarumizu Chuo Hospital, Tarumizu Municipal Medical Center, Tarumizu, Japan. ${ }^{4}$ Department of Nursing, Tarumizu Chuo Hospital, Tarumizu Municipal Medical Center, Tarumizu, Japan. ${ }^{5}$ Division of Clinical Laboratory, Tarumizu Chuo Hospital, Tarumizu Municipal Medical Center, Tarumizu, Japan.

Received: 14 January 2021 Accepted: 22 April 2021

Published online: 01 August 2021

\section{References}

1. Giske CG, Monnet DL, Cars O, Carmeli Y. Clinical and economic impact of common multidrug-resistant gram-negative bacilli. Antimicrob Agents Chemother. 2008;52(3):813-21. https://doi.org/10.1128/AAC.01169-07.

2. Roberts RR, Hota B, Ahmad I, Scott RD II, Foster SD, Abbasi F, et al. Hospital and societal costs of antimicrobial-resistant infections in a Chicago teaching hospital: implications for antibiotic stewardship. Clin Infect Dis. 2009;49(8): 1175-84. https://doi.org/10.1086/605630.

3. Akazawa T, Kusama Y, Fukuda H, Hayakawa K, Kutsuna S, Moriyama Y, et al. Eight-year experience of antimicrobial stewardship program and the trend of carbapenem use at a tertiary acute-care hospital in japan-The impact of postprescription review and feedback. Open Forum Infect Dis. 2019;6: ofz389. https://doi.org/10.1093/ofid/ofz389.

4. Hagiwara D, Sato K, Miyazaki M, Kamada M, Moriwaki N, Nakano T, et al. The impact of earlier intervention by an antimicrobial stewardship team for specific antimicrobials in a single weekly intervention. Int J Infect Dis. 2018; 77:34-9. https://doi.org/10.1016/j.ijid.2018.09.025.

5. Yamada K, Imoto W, Yamairi K, Shibata W, Namikawa H, Yoshii N, et al. The intervention by an antimicrobial stewardship team can improve clinical and microbiological outcomes of resistant gram-negative bacteria. J Infect Chemother. 2019;25(12):1001-6. https://doi.org/10.1016/j.jiac.2019.05.026.

6. Dellit TH, Owens RC, McGowan JE Jr, Gerding DN, Weinstein RA, Burke JP, et al. Infectious diseases society of America and the society for healthcare epidemiology of America guidelines for developing an institutional program to enhance antimicrobial stewardship. Clin Infect Dis. 2007;44(2): 159-77. https://doi.org/10.1086/510393.

7. Dyar OJ, Pagani L, Pulcini C. Strategies and challenges of antimicrobial stewardship in long-term care facilities. Clin Microbiol Infect. 2015;21(1):109. https://doi.org/10.1016/j.cmi.2014.09.005.

8. Maeda M, Muraki Y, Kosaka T, Yamada T, Aoki Y, Kaku M, et al. The first nationwide survey of antimicrobial stewardship programs conducted by the Japanese Society of Chemotherapy. J Infect Chemother. 2019;25(2):83-8. https://doi.org/10.1016/j.jiac.2018.11.001

9. Maeda M, Muraki Y, Kosaka T, Yamada T, Aoki Y, Kaku M, et al. Impact of health policy on structural requisites for antimicrobial stewardship: a nationwide survey conducted in Japanese hospitals after enforcing the revised reimbursement system for antimicrobial stewardship programs. J Infect Chemother. 2021;27(1):1-6. https://doi.org/10.1016/jijiac.2020.09.015.

10. Mas-Morey P, Valle M. A systematic review of inpatient antimicrobial stewardship programmes involving clinical pharmacists in small-to-mediumsized hospitals. Eur J Hosp Pharm. 2018;25(e1):e69-73. https://doi.org/10.113 6/ejhpharm-2017-001381.

11. Anderson DJ, Watson S, Moehring RW, Komarow L, Finnemeyer M, Arias RM, et al. Feasibility of core antimicrobials stewardship interventions in community hospitals. JAMA Netw Open. 2019;2(8):e199369. https://doi.org/1 0.1001/jamanetworkopen.2019.9369. 
12. Takito S, Kusama Y, Fukuda H, Kutsuna S. Pharmacist-supported antimicrobial stewardship in a retirement home. J Infect Chemother. 2020; 26(8):858-61. https://doi.org/10.1016/j.jiac.2020.04.008.

13. Miyoshi-Akiyama T, Tada T, Ohmagari N, Hung NV, Tharavichitkul P, Pokhrel $\mathrm{BM}$, et al. Emergence and spread of epidemic multidrug-resistant Pseudomonas aeruginosa. Genome Biol Evol. 2017;9(12):3238-45. https:// doi.org/10.1093/gbe/evx243.

14. Muraki Y. The role of pharmacists in antimicrobial stewardship. Yakugaku Zasshi. 2019; 139: 557-564 (Abstract in English).

15. Doernberg SB, Dudas V, Trivedi KK. Implementation of an antimicrobial stewardship program targeting residents with urinary tract infections in three community long-term care facilities: a quasi-experimental study using time-series analysis. Antimicrob Resist Infect Control. 2015;4(1):54. https:// doi.org/10.1186/s13756-015-0095-y.

16. Madaras-Kelly K, Jones M, Remington R, Caplinger CM, Huttner B, Jones B, et al. Antimicrobial de-escalation of treatment for healthcare-associated pneumonia within the veterans healthcare administration. J Antimicrob Chemother. 2016;71(2):539-46. https://doi.org/10.1093/jac/dkv338.

17. Storey DF, Pate PG, Nguyen ATT, Chang F. Implementation of an antimicrobial stewardship program on the medical-surgical service of a 100bed community hospital. Antimicrob Resist Infect Control. 2012;1 (1):32. https://doi.org/10.1186/2047-2994-1-32.

18. Hamada Y, Niwa T, Muraki Y, Aoyama S, Ueda H, Okudaira M, et al. A multicenter retrospective analysis regarding the clinical safety and efficacy of the post-approval meropenem dose in Japanese patients. Jpn J Chemother. 2015; 63: 560-567 (Abstract in English).

19. Gillbert DN, Chambers HF, Eliopoulos GM, Saag MS, Pavia AT. The sanford guide to antimicrobial therapy. Life Science publishing: Tokyo, 2019 (in Japanese).

20. The JAID/JSC guide to clinical management of infectious disease/guidelinepreparing committee: The JAID/JSC guide to clinical management of infectious disease. Life Science publishing: Tokyo, 2019 (in Japanese).

21. Waters CD. Pharmacist-driven antimicrobial stewardship program in an institution without infectious diseases physician support. Am J Health-Syst Pharm. 2015;72(6):466-8. https://doi.org/10.2146/ajhp140381.

22. Gentry CA, Greenfield RA, Slater LN, Wack M, Huycke MM. Outcomes of an antimicrobial control program in a teaching hospital. Am J Health-Syst Pharm. 2000;57(3):268-74. https://doi.org/10.1093/ajhp/57.3.268.

23. Gums JG, Yancey RW Jr, Hamilton CA, Kubilis PS. A randomized, prospective study measuring outcomes after antibiotic therapy intervention by a multidisciplinary consult team. Pharmacotherapy. 1999;19(12):1369-77. https://doi.org/10.1592/phco.19.18.1369.30898.

\section{Publisher's Note}

Springer Nature remains neutral with regard to jurisdictional claims in published maps and institutional affiliations.

Ready to submit your research? Choose BMC and benefit from:

- fast, convenient online submission

- thorough peer review by experienced researchers in your field

- rapid publication on acceptance

- support for research data, including large and complex data types

- gold Open Access which fosters wider collaboration and increased citations

- maximum visibility for your research: over $100 \mathrm{M}$ website views per year

At $\mathrm{BMC}$, research is always in progress.

Learn more biomedcentral.com/submissions 